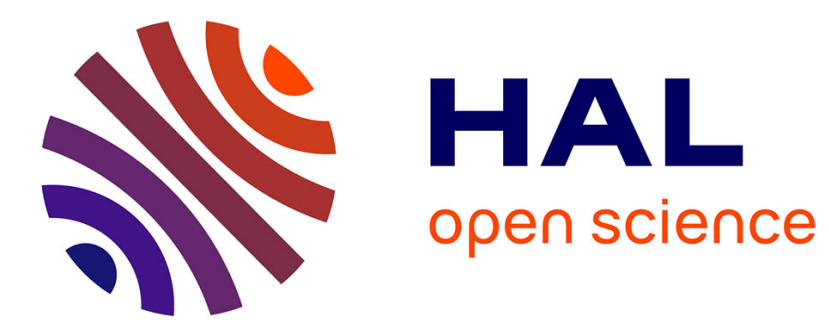

\title{
The Meaning of the Opposition Between the Healthy and the Pathological and its consequences
}

\author{
Maël Lemoine
}

\section{To cite this version:}

Maël Lemoine. The Meaning of the Opposition Between the Healthy and the Pathological and its consequences. Medicine, Health Care and Philosophy, 2009, 12 (3), pp.355-362. 10.1007/s11019-0089163-x . halshs-00792410

\section{HAL Id: halshs-00792410 https://shs.hal.science/halshs-00792410}

Submitted on 3 May 2013

HAL is a multi-disciplinary open access archive for the deposit and dissemination of scientific research documents, whether they are published or not. The documents may come from teaching and research institutions in France or abroad, or from public or private research centers.
L'archive ouverte pluridisciplinaire HAL, est destinée au dépôt et à la diffusion de documents scientifiques de niveau recherche, publiés ou non, émanant des établissements d'enseignement et de recherche français ou étrangers, des laboratoires publics ou privés. 


\section{The Meaning of the Opposition between the Healthy and the Pathological and its Consequences}

Maël Lemoine (University of Tours)

\section{Introduction}

Within the class of human biological phenomena, medical common sense, the philosophy of medicine and medical science oppose healthy and pathological phenomena in such a way that no phenomenon can be healthy and pathological at the same time, and no phenomenon can be neither healthy nor pathological. This is the kind of certitude which is justified by intuition alone; in no way can it be justified by any exhaustive observation of biological phenomena or by demonstration. Is it, though, at least possible to clarify the logical structure of this opposition so as to provide some elements of knowledge about this crucial distinction for medical science?

\section{A logical analysis of the 'opposition' between healthy and pathological.}

Provided that

(A1) every biological phenomenon is either healthy or pathological,

meaning also that

(A2) no biological phenomenon is both healthy and pathological at the same time,

how then should the healthy and the pathological be contrasted, that is, by what type of opposition? This opposition may be construed at least in 3 different ways, that is, 1) as a disjunction of propositions ('it is the case either that $x$ is healthy or that $x$ is pathological'), 2) as a separation into sets (' $x$ belongs either to the set of healthy or to the set of pathological phenomena'), 3) as the 
exception of a fact (' $x$ is pathological') to a norm (' $x$ should be healthy').

As a disjunction of propositions $(h \mathrm{~V} p)$, the opposition means that either ' $x$ is healthy' $(h)$ or ' $x$ is pathological' $(p)$ is the case. Yet what is the nature of this disjunction? If it is an intensional disjunction, that ' $\mathrm{x}$ is healthy' is not the case materially implies that 'x is pathological' is the case $(\neg \mathrm{h}$ $\rightarrow \mathrm{p}$ ), and vice versa: this is something that we know from the nature of the actual world. On the other hand, if it is an extensional disjunction, we know by purely logical consideration that it is true, and that it must be true, in all possible worlds. The implication is, in this case, a strict implication (Gabbay and Guenthner, 1994, II, p. 4-5).

As a separation into sets $(\{h / p\})$, the opposition of the healthy and the pathological means that 'every biological phenomenon that does not belong to the set of healthy phenomena belongs to the set of pathological phenomena' (and vice versa). This separation too has a twofold interpretation. The set of everything that is in $h$ and the set of everything that is not in $p$ are actually one and the same set (and vice versa). In an extensional interpretation of the definitions of the healthy and the non-pathological, this truth implies that there is one and only one definition of both terms, therefore, one and only one definition of the terms 'healthy' and 'pathological'. On the other hand, according to an intensional interpretation of these definitions, although the set happens to be the same, the terms 'healthy' and 'non-pathological' may be defined differently, as may the terms 'healthy' and 'pathological'. For instance, although it may be the case that all the dead people in Chambord City can be found buried in the graveyard on April $29^{\text {th }}$, and conversely that everyone buried there is dead, yet 'dead in Chambord City on April $29^{\text {th' }}$ and 'buried in the graveyard in Chambord City on April $29^{\text {th' }}$ cannot be defined in the same terms (Kneale \& Kneale, 1962, p. 618628).

As a norm, 'x should $h^{\prime}$ (where ' $h$ ' stands for 'be healthy' and ' $p$ ' for its opposite, 'be pathological') implies either that ' $x$ should-not $p$ ' or that ' $x$ should not- $p$ '. That $x$ should-not $p$, means that no norm demands that $x$ be non-pathological, even though a norm demands that $x$ be healthy; 
on the other hand, that it should not- $p$, means that the same norm demands both that $x$ be healthy and that $x$ be non-pathological. The negation of the 'should' is an external negation, whereas the negation of the ' $p$ ' is an internal negation (Horn, 2001, p. 363-365; for similar applications in deontic logic, see p. 134). If $h$ is opposed to $p$ as a norm to its external negation, then ' $x$ should $h^{\prime}$ does not imply that ' $x$ should not- $p$ ', that is, $h$ is normal, but $p$ is not abnormal. On the other hand, if $h$ is opposed to $p$ as a norm to its internal negation, then ' $x$ should $h$ ' does indeed imply that ' $x$ should not- $p^{\prime}$, that is, $p$ is abnormal precisely because $h$ is normal. In the former case, the normal is opposed to the (not-normal) factual; in the latter case, it is opposed to the abnormal.

Subtle though these distinctions may appear, they are crucial for the present problem. For there seem to be two and only two different positions: one may either assume that the healthy and the pathological are natural opposites, needing two definitions, and contrast as normal and not normal, or that they are logical opposites, needing only one definition, and contrast as normal and abnormal. To avoid further technicalities here, I must ask the reader to accept, on the basis of its prima facie plausibility, the fact that in the present case at least, intensional disjunction, intensional definition and external exception stem from the same coherent point of view, as do extensional disjunction, extensional definition and internal exception.

Thus two mutually exclusive opposites that together compose the whole domain of quantification (Gabbay \& Guenthner, I, p. 36), such as 'healthy' and 'pathological', may be either termed factual (that is, intensional and external) or rational (that is, extensional and internal) opposites. How does this distinction between oppositions clarify the meaning of the opposition between the healthy and the pathological? It will be shown that this same ambiguity - is it a factual or a rational opposition ? - is the main obstacle to either a philosophical or a scientific elucidation of the nature of this opposition of the healthy and the pathological.

\section{A philosophical analysis of the opposition of the healthy and the pathological.}


One can tell philosophical definitions from commonsense definitions by the univocal meaning they try to give to terms. They succeed in doing this either by impoverishing and restricting the field of usual meanings, by articulating different meanings in an hierarchical or deductive order, or by creating new meanings that did not exist before in common use of language. Does philosophical analysis make it possible to choose between factual and rational opposition by defining the healthy and/or the pathological? 3 possibilities come to mind: either defining 'healthy' as 'non-pathological' (Boorse, 1977), defining 'pathological' as 'non-healthy' (Nordenfelt, 1995), or defining the healthy and the pathological as contraries (Canguilhem, 1966).

a. 'healthy' as 'non-pathological'.

"It is a traditional axiom of medicine that health is the absence of disease. What is a disease? Anything that is inconsistent with health. If the axiom has any content, a better answer can be given. The most fundamental problem in the philosophy of medicine is, I think, to break the circle with a substantive analysis of either health or disease." (Boorse, 1977, p. 542) To do that, Boorse changes this circular definition by means of a spiral series of definitions. Thus, each time the circle threatens to close up on itself in a reciprocal definition of health and disease, a change of level makes it possible to define a new term. Thus, departing from a biological conception of 'health' as a "normal functional ability" (id., p. 555), a medical theoretical conception of disease is obtained by privation: disease as "a type of internal state which is either an impairment of normal functional ability, i.e. a reduction of one or more functional abilities below typical efficiency, or a limitation on functional ability caused by environmental agents." (id., p. 567) Then one moves towards a negative concept of health, in a medical theoretical sense, as "absence of disease" (id.). Further on, illness in the practical sense is the privation of health (in the negative sense), that is, "a disease serious enough to be somewhat incapacitating, which thereby supports normative judgments about treatment and responsibility" (id., p. 552); hence a definition of health in the practical sense as "the 
absence of treatable illness" (id., p. 542). Beyond this point, whenever one considers that an individual is not in the state of not having to be treated, a negative value-laden conception of disease becomes possible, as well as a positive valuative conception of health as "not one ideal, but a kind from which various ideals may be selected and pursued", whose pursuit "forces a choice between incompatible excellences" and requires a "value judgment" (id., p. 571).

Each of these four disjunctions is extensional, that is, rationally necessary in all possible worlds. A biological phenomenon functions or dysfunctions, is present or absent, treatable or intractable, valuable or worthless. On the other hand, it is not rational, but factual, for instance, that a medical-theoretical concept of disease is opposed to to a biological concept of health: in this respect each disjunction is intensional, that is, actually necessary in this world for purely natural reasons.

The same ambiguity undermines the separation of the two sets of phenomena. Each of these four pairs of opposites is also extensionally defined, that is, by one (and one only) of the two sets: when one member of the pair is defined, the definition of the other rationally follows. But on the other hand, how could one deduce from the mere negation of the fact that something is 'healthy' (biologically speaking), that it is medically pathological? For the different sets of the pathological are not necessarily one and the same. Therefore, these separations appear to be also intensional.

According to Boorse, statistical normality alone is not enough to define health (id., p. 546); hence it must be defined as the statistical normality of biological functioning. For the normal is not merely the frequent, it is also the natural (id., p. 554), and the pathological is therefore that which fails to be natural. That is, the pathological is not something that is external to the norm - what should-not be -, but something that is internal to the norm - what it says should not-be. In other words, everything which is not normal and healthy, i.e. which does not conform to the biological standards of the species, is abnormal and pathological. It is therefore a rational opposition. On the other hand, 'the natural' is defined by "typological and teleological notions" (id., p. 554), that is, the 
"goals" of the organism, defined without referring to any values, from the mere objective standpoint of the observation in each science (id., p. 556), together defining a "species design, i.e. the typical hierarchy of interlocking functional systems that supports the life of organisms of that type" (id., p. 557). But from this viewpoint, no biological phenomenon is either healthy or pathological, normal or abnormal: for if the healthy is defined as the conformity of biological functioning to this "species design”, the pathological is defined at times privatively as everything that opposes such biological functioning (id., p. 559, 567, passim), and at others contrarily as everything that has veered off course from this functioning (id., p. 543). In the former case one may, for instance, consider a mole not to be pathological, for it does not in any way oppose normal biological functioning, whereas in the latter case a mole is pathological, for it does not pursue the standard typical goal of the species. The same goes for the evolution of a species: a genetic mutation that gives birth to a viable living thing whose biological functioning is different must be considered to be either a pathological case (defined rationally as abnormal) or a non-pathological case (defined factually as not-normal).

In spite of his intention to define health as the rational opposite of disease, Boorse is forced to consider the healthy and the pathological to also be factual contraries, each being defined separately, each constituting a natural phenomenon.

\section{b. 'pathological' as 'non-healthy'.}

Nordenfelt emphasizes the first two aspects of the rational opposition at the same time: the conceptual (or rational), and not merely empirical (or factual) characteristic of the opposition of health and disease, and the necessity that the one be defined by the other, but not vice versa (Nordenfelt, 1995, pp. 13-4). If there were an opposition between two separately defined terms, he stresses, it would not have a conceptual character anymore and would be a mere empirical opposition. It would not then be logically impossible that health and disease coexist, i.e. that some diseases do not alter health. According to Nordenfelt, the only first concept that has to be defined 
positively is health, and not disease (as Boorse claims). " $A$ is healthy if, and only if, $A$ has the (...) ability, given standard circumstances, to realize all the goals necessary for his minimal happiness" (id. p. 148). Thus defined, health is the state that should be, not according to biological, but to social, cultural or ethical norms. As "non-health" (id. p. 105), illness is the internal exception of this norm, that is, what social, cultural and ethical norms define as what should not-be.

But not every illness is pathological in the medical sense. Among the different states of "disability" (id. p. 35) which a subject may be affected by, and which prevent him from reaching one of the goals defined above, maladies are "internal entities to a person's body or mind which tend to impair his health" (id. p. xvi), a category which includes diseases, but also injuries or congenital defects (id. pp. 108-109), and a disease is a "bodily or mental process which tends to compromise health" (id. p. 108). Only those illnesses that are maladies (or even, to some, those that are diseases) are pathological. Illness is therefore pathological only as this particular state of illbeing which a malady (or a disease) sometimes produces. That is to say, the disjunction and the definition of health and illness are intensional and rational, in the same way as the exception of illness to health is internal and rational. But pathological states or phenomena, that is, maladies or diseases, happen to be factors (among others) that impair health, so that the disjunction and the definition of pathological and healthy phenomena is only intensional, and the exception of the pathological to the norm of the healthy, only external, for a social norm cannot internally define precisely which kind of biological phenomena impairing health must be considered as pathological. Necessity is not clear-cut, the separation of sets is blurred, and it will not always be explicit whether a particular condition should not-be, even when it is explicit that it should-not be. On the one hand, Nordenfelt sticks to a rational opposition of health and illness, but on the other hand, he shifts to a factual opposition of the healthy and the pathological.

c. the healthy and the pathological as contraries. 
Georges Canguilhem's views about the philosophy of medicine are not well-known in contemporary international philosophical literature, partly because of the peculiarities of French idiomatic expressions, partly because of the complexity of the design of his masterpiece, Le normal et le pathologique. Canguilhem rejects any privative definition of the healthy and the pathological. The pathological is indeed what one necessarily notices before conceiving of anything like health, which nevertheless preexists it, though now compromised and limited; but this does not mean one considers it to be the mere privation of disease (Canguilhem, 1966, p. 52). In fact, since the XIXth century, a certain physiological thesis has conceived of the pathological as a mere quantitative variation of the healthy, that is, neither a mere privation nor a qualitative difference. Against this conception, Canguilhem emphasizes the qualitative aspect of this opposition, which would become meaningless if it were to be reduced to a mere quantitative difference, though it can indeed be adequately expressed by such a means (id., p. 67): what is far above the norm cannot be understood as an abnormal variation but in the perspective of possible bad consequences for the patient, that contrast a given biological functioning with another on the basis of their respective natures. Once the pathological has been distinguished from the abnormal in this manner, it is not necessary to define the pathological as the privation of the healthy, or vice versa. On the contrary, it remains to define disease as a biologically negative value, that is, something a living being runs away from (id. p. 118), and, separately, to define the healthy as something a living being seeks after. Thus the healthy and the pathological are neither disjuncted, nor defined, internally, if the former is referred to the concept of a "privileged behavior" and the latter to the concept of "catastrophe reaction" that Kurt Goldstein introduced (id. p. 121). As contraries, the healthy and the pathological quite resemble two natural phenomena, the opposition of which does not imply logical necessity. "The contents of the pathological state cannot be deduced from the contents of health, except for a difference of size: disease is not a variation in the dimension of health; it is a new dimension of life." (id. p. 122). The healthy and the pathological therefore seem to be opposite directions of life: 
two divergent orientations, two contradictory ways of living, two different "paces of life". On the one hand, diseased life orients negatively towards the avoidance of catastrophe reactions (id. p. 132); on the other hand, healthy life orients positively towards the creation of a privileged behavior, that is towards the creation of new norms which the organism lays down on its environment (this is what Canguilhem calls "normativité", pp. 132-133). This opposition of directions, i.e. this contrariety, can incidentally be expressed by quantitative variables, though not always numerically, but at least hierarchically, even by ordinals, through the inclusion of the "inferior" (id. p. 120) pathological norm within the healthy, superior norm: "a norm of life is superior to another when the former comprises both what the latter allows and what it forbids" (id. p. 119). In the pathological state, the organism only orients towards preserving its equilibrium with the environment in which it survives, whereas in the healthy state, it is characterized by "the possibility of overcoming the norm that defines the momentary normal, the possibility of tolerating breaches of the habitual norm and instituting new norms in new situations" (id. p. 130).

It is absolutely clear that health is a positive fact, the fact of "the initial indetermination of the capacity to institute new biological norms" (id. p. 129), or rather, the "safety" (id. p. 131) margin or "tolerance for the infidelities of the environment" (id. p. 130). Yet it is less evident in the case of disease. "Conversely, the distinctive feature of disease is that of being a reduction of the tolerance to the infidelities of the environment" (id. p. 132). Even if a reduction of the tolerance is not merely a quantitative, numerical diminution, it is indeed a privative concept all the same, different by nature from the phenomenon of avoiding a catastrophe reaction, by which Canguilhem first defined the pathological. For the exclusive orientation of the organism towards such an avoidance does not indeed imply any reduction of tolerance, and the same is true of the reciprocal sentence. A living being can focus on avoiding ill-treatment without being diminished. Here again, then, there are two conceptions of the opposition of the pathological to the healthy, as rational, and as factual. On the one hand, when they are defined as contraries, the healthy seems to be a 
normative (not normal) process of self-affirmation and creation, and the pathological seems to be a process of avoidance (id. pp. 155-156); on the other hand, when they are defined as rational opposites, the healthy seems to be an initial indetermination, a safety margin or a functional overabundance of its vitality, and the pathological a determination and reduction of this safety margin, and a strict functional adaptation in a survival environment. Canguilhem cannot hold to an opposition of contraries, as he first intended to do, because such a contrariety of directions or tendencies of the living cannot exclude the logical possibility of a brand new third orientation, neither healthy nor pathological, which would be neither the creation of a privileged behavior, nor the avoidance of panicky states or catastrophe reactions. Hence the necessity to also define the pathological as a privation of the healthy: for if a living being must be either overabundant or strictly adapted to a (reduced) environment, the opposition then seems to be a rational necessity, the healthy then looks like the normal or expected phenomenon, and the pathological is defined merely on this basis.

Every philosopher who has been concerned by this question of defining the healthy and the pathological seems to have emphasized that it is fruitless to define one without the other, but they have all hesitated between two possibilities. It remains to be seen whether a scientific use of these terms can clear up this ambiguity, or whether it is bogged down with it.

\section{Can some elements of medical science contribute towards a definition of the logical nature of the opposition?}

The definition of a scientific term wavers between an ontological and an operating perspective. In an ontological perspective, to define is above all to indicate a fact in order to clarify what this fact is, without sticking specifically to any of the many possible criteria which make it possible to claim its existence: thus 'this is $\mathrm{H}_{2} \mathrm{O}$ ' is a same fact that can be confirmed by many ways. On the other hand, in an operating perspective, defining consists in indicating a fact as that whose 
existence is confirmed by one criterion and only one, so that changing that criterion might be tantamount to defining a different fact: thus a certain subatomic particle may be defined by the only process that proves its existence, so that successfully using any other criterion cannot guarantee one is observing the same particle. The ontological perspective therefore guarantees the identity of the fact against all possible mistakes in applying the criterion, but may be equivocal, for nothing can ensure that what seems to be the same fact, proved by successive identical tests or different criteria, is not actually various initially mixed-up facts. On the other hand, the operating perspective guarantees the identity of what is defined against the multiplicity of tests, but not against possible errors in the application of the criterion. By adopting an ontological perspective, medical analysis contrasts the presence of the criterion of a disease with the presence of the disease itself. Even in the case of a reference criterion, a gold standard, and even in the case of a univocal (pathognomonic) sign, this distinction accounts for the theoretical possibility that the diagnosis be established otherwise by a different criterion, or that the sign be either ill-observable or ill-observed. On the other hand, in an operating perspective, the physician considers disease as what is present whenever the condition of the gold standard is fulfilled: for lack of any other direct access to the fact of a disease, it is often necessary to define it arbitrarily by the presence of a sign that is considered to be the criterion of truth. Bernard Grenier thus clearly contrasts the truth of a disease with the presence of a disease. "The gold standard defines an identifiable 'truth', he says, i.e. that of the presence of the disease (...) if the criteria of evidence are certified, or that of its absence otherwise." (Grenier, 1999 p. 18; Zweig et al., 1993)

Criteria, for instance the gold standard of a disease, have the property of making clear which situations 'are the case' and which 'are not'. The discrimination it establishes on the level of the judgmental must therefore ideally correspond to a difference on the level of the factual, whose existence is evident. This judgmental discrimination is ideally established whenever a 'perfect separator' exists, which assumes a parallel perfect separation in the facts. But in the case of an 
imperfect separator, one often assumes in medicine that the criterion is what is ill-defined (on the operating level), without questioning the very principle of a factual difference on the ontological level. In other words, one assumes that there actually is a perfect difference between those who are affected by a disease $D$ and those who are not, and that the criterion that makes it noticeable is not absolutely efficient. This is the meaning of the distinction between 'true positive' and 'false positive', that is, positive according to a test which either should or should not qualify a case as positive from the viewpoint of a fact that cannot be known except through a gold standard that is not the disease itself. This perfect separation in the factual may be stated in the form of the following axiom: 'Given any disease $D$, the population divides between those who are affected by $D$ and those who are not affected by $D$, it being impossible that anyone be neither affected nor non-affected by $D$.' This is indeed an axiom, because it deals clearly with the conceptual necessity of a postulated truth, not the factual or natural necessity of a physiological law, or the universality of an observed fact. For in order to prove that there is indeed a perfect ontological separation between diseased and nondiseased, a perfect operating separator would be required. How can it be proven that an operator is perfect, if it cannot by definition be compared with another criterion of the same reality? For any given disease, the healthy population is therefore defined by the absence of the disease, identified more or less perfectly by the absence of the sign, whereas the pathological population is defined by the presence of the disease, identified more or less perfectly with the presence of the sign. All the conditions are fulfilled to allow one to consider this opposition to be that of possession (D+) and privation (D-), that is, a rational opposition. If the healthy and the pathological were contraries, that is, in a factual opposition, they would be identified by the presence of different signs that happen never to be present together in the same individual at the same time. There would be signs of D+ and signs of D-. The gold standard which is defined by the conjunction: $s i g n_{D+}, \operatorname{sign}_{D-}$, would define a perfect separation provided that all individuals affected by $D$ had $\operatorname{sign}_{\mathrm{D}+}$ and not one of them had $\operatorname{sign}_{\mathrm{D}-}$, whereas all individuals free from $D$ had $\operatorname{sign}_{D-}$ and not one of them had $\operatorname{sign}_{D+}$. 
The axiom therefore forces us to attribute to the imperfection of a criterion what may be a matter of a factually complex state. Hence procedures of assessment of the uncertainty of a diagnosis that tends only to solve criteriological problems. If a physician is sometimes right, sometimes wrong when considering a given patient to be diseased on the basis of the presence of certain signs, this is above all the case because this sign is not a perfect indicator of the presence of the disease. The best of all possible indicators is considered to be the gold standard: "the procedure that establishes the patient's true disease status is referred to as a gold standard" (Zhou et al., 2005, p. 600). Yet if the gold standard is indeed a reference test, it has nevertheless its own sensitivity and its own specificity, which constitute references in the assessment of the sensitivity and specificity of other diagnostic tests. However, most gold standards do not have a perfect sensitivity or specificity, i.e., have a sensitivity and a specificity that do not equal 1 , that is, they do not identify all cases of $D+$ and do not eliminate all cases of $D$-; one may even say: in principle, they do not. For the ontological perspective of the scientific definition of a pathology forces one to make a difference, out of cautiousness, between disease as an actual state and disease as the positive result of a test. It becomes necessary then to assess the discriminatory performance of the test. One such paradigm, corresponding to this definition, is the 'ROC analysis' paradigm in medicine, where ROC stands for: Receiver-Operating Characteristic (Zweig et al., 1993). This paradigm binds together the specificity and the sensitivity of a test when the separating line between the normal and the pathological is displaced. Every analysis of a sign must be defined and assessed according to this paradigm. As the ontological axiom: 'any patient is either D+ or D-' is not being questioned, the uncertainty is therefore always attributed to the measurement or to the test procedure itself. The whole question thus seems to be the search for a solution to the various problems formulated once this axiom is assumed: for instance, the discrimination may be binary (D+/D-), non-binary (Feinstein, 1990; Simel et al., 1992; Mossman, 1999; Coste and Pouchot, 2003), nominal, ordinal and even continuous (Obuchowski, 2006); all the problems come down to the same type of solution: 
finding a way to get back to this ROC curve. Even in cases where there is no gold standard, and where the specificity and sensitivity of the tests in use are therefore unknown (Enøe et al., 2000; Hui and Walter, 1980; Walter and Irwig, 1988; Hui and Zhou, 1998; Zhou et al., 2005), the uncertainty must be expressed as the uncertainty of a criterion, but not as the qualitative complexity of a biological situation: it must be the uncertainty of a status, not the complexity of a state. The same goes for every case where the separation between $\mathrm{D}+$ and $\mathrm{D}$ - is not made by a variable, but by an interval which is often called a 'grey zone', that is a range of values that cannot determine whether the pathology is present or absent (Coste and Pouchot, 2003 p. 304), and for every case where using the gold standard produces errors through imprecision (which is known as the 'fuzzy gold standard'), leads to an uncertain discrimination of the healthy and the pathological states, and makes the assessment of a diagnostic test more difficult, whether the error is statistically dependent or not (Phelps and Hutson, 1995). In such cases, one generally attributes this fuzziness and uncertainty either to the difficulty of a measurement that involves a high risk of error, as is the case as in the interpretation of a X-ray, or to biological variability, which results in the test being adequate only for the majority of cases that occur (Walter and Irvig, 1988 pp. 923-924). Yet a different possible cause of this fuzzy status cannot be ruled out: the imperfection of the difference between healthy cases and pathological ones in factual reality, that is a factual opposition which actually corresponds to a contrariety rather than a rational opposition between possession and privation. However this possibility is effectively ruled out in the assessment of the uncertainty of a test whose specificity and sensitivity are measured.

There is a particular fact which leads one to challenge this prejudice in favour of the rational opposition between privation and possession (of health, of a disease). 'Being D+' or 'being D-' must actually be understood in relation to a chosen pathology and not in absolute terms. The fact this definition of a pathological state addresses is therefore not so much a biological state as an aspect of this state: the particular aspect which the observer's question highlights, for instance, the 
fact of having or not having sores, being HIV+ or not, and so on. In determining a pathological state, the factual plays only a minor part, the physician's intentional aim playing the major part: it is less important to know the patient's state than to answer 'yes' or 'no' to a question, that may indeed be badly put. Thus the specificity of a test must be measured in relation to a selected population whose assumed state is D-, but to which the application of the test must be also pertinent (Grenier, 1999 p. 21). For the D- class is not the whole group of those who are not in the pathological state in question, but a subclass of individuals effectively circumscribed by a more specific supposition, for instance, the supposition of a risk factor. On the other hand, signs are seldom perfectly specific, each generally referring to various possible pathologies. The clinician's intentional aim puts this or that pathology in perspective, and considers this or that sign to be a test of its presence. The formalization of this situation through a series of interpretations of the same test in various possible perspectives, as proposed in Obuchowski et al., 2001, seems to solve the problem of the arbitrary or subjective choice of the perspective on the pathologies to be tested for. It remains that, each time, it is the expectation of a defined state that makes one consider its absence to be a privation, not the state itself. Yet, even if many pathological signs are thus negatively defined as the privation of a normal or expected phenomenon (constipation, amenorrhea, insomnia, anencephaly, dyslipidemia, and so on), some other signs, on the other hand, seem to be constitutive of contrary states. Cyanosis is not merely the privation of the normal coloring of the skin, but rather one of the many colors the skin can have; croup is not a privation, but one of the possible alterations of the state of the larynx, and so on. Drawing a clinical picture from the presence or absence of numerous given signs, is like trying to reproduce each hue of a maestro's palette by means of the opposition between two primary colors.

As soon as one considers the opposition between the healthy and the pathological to be a binary alternative, one considers it to be conceptual or rational, and as soon as one considers it to be 
conceptual, one must acknowledge the possibility that it does not always apply exactly to reality. Conversely, as soon as one begins by considering a real opposition between states that actually exclude each other reciprocally, it is no longer possible to guarantee there can only be two possibilities. As a matter of fact, the assessment of a test becomes a lot more difficult, but one can bet that statisticians', clinicians' and epidemiologists' imaginations can formalize a factual opposition as well as a rational opposition. Only experiments can show whether it would be useful to consider healthy and pathological states to be two possible states instead of considering them to be the only two possible states.

\section{Conclusion. Practical outcomes of the logical problem of the opposition between the healthy} and the pathological.

Through statistical means, experimental results must, then, divide phenomena into two groups: pathological and normal. The division depends on these results and the separator is not determined by purely mathematical laws. Yet the principle of the division is not a physiological law either: the (quantitative) threshold or the nature of the (qualitative) criteria one considers to be the separator is determined in an experimental manner, but one theoretically decides that there is a separator and two classes. This has several outcomes according to the nature of the criterion:

1. when it is a qualitative criterion, such as the presence or absence of an observable sign of D, the clinician is confronted with the possibility of dubious or uncertain signs. At bottom, the reason is that there is no real or actual state of privation of a sign. Privation is the property of a clinical judgment, not of a biological state. Actual properties may be contraries and exclude one another as is the case with sight and blindness, but nothing will ever prove the necessity that these are the only two possible qualities of the function. They are only in the limited scope of a determined question. 
2. When it is a quantitative criterion, be it numerical and continuous, discontinuous or merely ordinal, the problem is that of determining the threshold, and of dealing with a possible grey zone. Canguilhem emphasized that there is neither 'too much...' nor 'not enough...' of the state per se, i.e. without any qualitative reference criterion of discrimination between the healthy and the pathological. The problems of the 'arbitrary' determination of the threshold and of grey zones therefore come from the originally qualitative nature of the opposition.

3. When it is a prognostic criterion, that is, a discrimination according to a taken risk, the problem is yet again one of the arbitrary threshold (of the risk). Oversimplifying the possible evolution of a physiological state by expressing it as significant or not significant risks of D, means a complex biological fact is reduced to a mere logical formula: the present state $S$ may actually evolve into $S_{1}, S_{2}, S_{3}, \ldots S_{n}$, and not only into $\mathrm{D}(x)+$ or $\mathrm{D}(y)$-.

4. When it is a pragmatical criterion according to which one recommends or advises against a particular therapy, the problem comes down to the multiplicity of qualitative and quantitative criteria one needs to aggregate to determine a decision. This, for instance, is the case in the assessment of the quality of life through given items. Here again, the problem lies in trying to render a qualitative multiplicity of contraries into a binary opposition - to treat or not to treat.

Lastly, the conceptual characteristic of the opposition between the healthy and the pathological is in perpetual tension with the evolutionary aspect of scientific knowledge. Whatever the novelty of discoveries and the complexity of explanations, one will always have to reduce them to a simple binary polarization. The therapeutic prospect requires one 1) to maintain this conceptual unity of the set of pathological phenomena as opposed to the set of healthy phenomena, along with the conceptual impossibility of any intermediate phenomena (neither...nor...);2) to define each of them separately, yet to consider that their definitions are logically bound together; 3 ) to consider 
that the exception of the pathological to the norm of the healthy is both a mere case that does not fit, and at the same time, is always circumscribed as that which contradicts the norm. Yet the stupendous variety of diseases probably condemns this demand for conceptual unity of factual reality to being imprecise and to producing ineffectiveness.

\section{References}

Boorse, C.: 1977, 'Health as a theoretical concept', Philosophy of Science 44, 542-573.

Canguilhem, G.: 1966, Le normal et le pathologique. Paris: Presses Universitaires de France.

Coste J., Pouchot J., 2003: 'A grey zone for quantitative diagnostic and screening tests', International Journal of Epidemiology 32, 304-313.

Enøe C., Georgiadis M. P., Johnson W. O., 2000: 'Estimation of sensitivity and specificity of diagnostic tests and disease prevalence when the true disease state is unknown', Preventive Veterinary Medicine 45, 61-81.

Feinstein A. R., 1990: 'The inadequacy of binary models for the clinical reality of three-zone diagnostic decisions', Journal of Clinical Epidemiology 43, 109-113.

Gabbay D., Guenthner F. (ed.), 1994. Handbook of Philosophical Logic. Vol. I: Elements of Classical Logic. Vol. II: Extensions of Classical Logic. Dordrecht / Boston / London: Kluwer Academic Publishers.

Grenier B., 1999, Evaluation de la décision médicale. Introduction à l'analyse médico-économique (3rd ed.). Paris: Masson.

Horn L. R., 2001. A Natural History of Negation. Chicago: University of Chicago Press. Reissued CSLI Publications.

Hui S. L., Walter S. D., 1980: 'Estimating the error rates of diagnostic tests', Biometrics 36, 167171.

Hui S. L., Zhou X. H., 1998: 'Evaluation of diagnostic tests without gold standards', Statistical 
Methods in Medical Research, 7, 354-370.

Kneale W. \& Kneale M., 1968. The Development of Logic. Oxford: Clarendon Press.

Mossman D., 1999: 'Three-way ROCs', Medical Decision Making 19, 78-89.

Nordenfelt, L.: 1995, On the Nature of Health. An Action-Theoretic Approach, Dordrecht/Boston/London: Kluwer Academic Publishers.

Obuchowski N.A, Goske M.J., Applegate K.E., 2001: 'Assessing physicians' accuracy in diagnosing pediatric patients with acute abdominal pain: measuring accuracy for multiple diseases', Statistics in Medicine 20, 3261-3278.

Obuchowski N.A, 2006: 'An ROC-type measure of diagnostic accuracy when the gold standard is continuous-scale', Statistics in Medicine, 25, 481-493.

Phelps C. E., Hutson A., 1995: 'Estimating diagnostic test accuracy using a 'Fuzzy gold standard", Medical Decision Making, 15 (1), 44-57.

Simel D. L., Matchar D. B., Feussner J. R., 1992: 'Diagnostic tests are not always black or white : or, all that glitters is not [a] gold [standard]', Journal of Clinical Epidemiology 44, 967-71.

Walter S. D., Irwig L. M., 1988: 'Estimation of test error rates, disease prevalence and relative risk from misclassified data : a review', Journal of Clinical Epidemiology 41 (9), 923-37.

Zhou X.-H., Castelluccio P., Zhou C., 2005: 'Nonparametric estimation of ROC curves in the absence of a gold standard', Biometrics 61, 600-609.

Zweig M.H., Campbell G., 1993: 'Receiver-operating characteristic (ROC) plots - a fundamental evaluation tool in clinical medicine', Clinical Chemistry, 39, 561-577. 